\title{
Dynamical instabilities of microwaves generated with optoelectronic oscillators
}

\author{
Y. Kouomou Chembo, ${ }^{1,2, *}$ Laurent Larger, ${ }^{1}$ Hervé Tavernier, ${ }^{1}$ Ryad Bendoula, ${ }^{1}$ \\ Enrico Rubiola, ${ }^{1}$ and Pere Colet $^{2}$ \\ ${ }^{1}$ UMR CNRS FEMTO-ST 6174, Département d'Optique P. M. Duffieux, Université de \\ Franche-Comté, 16 Route du Gray, 25030 Besançon cedex, FRANCE \\ ${ }^{2}$ Instituto Mediterráneo de Estudios Avanzados IMEDEA (CSIC-UIB), \\ Campus Universitat de les Illes Balears, E-07122 Palma de Mallorca, SPAIN \\ *Corresponding author: ckyanne@imedea.uib.es
}

We introduce a time-domain model to study the dynamics of optoelectronic oscillators. We show that due to the interaction between non-linearity and time delay, the envelope amplitude of ultra-pure microwaves generated by optoelectronic oscillators can turn to be unstable. Our analytical predictions are confirmed by numerical simulations and experiments. (c) 2007 Optical Society of America

OCIS codes: $120.3940,190.3100,350.4010$. 
Recent advances in ultra-stable microwave oscillations have introduced novel architectures referred to as optoelectronic oscillators (OEOs) [1]. Typically, single-mode OEOs are able to produce radio-frequency oscillations with extremely high spectral purity in the microwave range at up to tens of $\mathrm{GHz}$, with sideband phase noise levels as low as $-160 \mathrm{dBrad}^{2} / \mathrm{Hz}$ at $10 \mathrm{kHz}$ from the carrier. This performance is achieved through the use of an unusual energy storage principle, based on a long optical fiber delay line instead of the classical concept of resonators. OEOs are therefore candidates for various applications in spatial, lightwave and radar technologies.

However, theoretical description of these systems has been done only through the implicit asumption of a stationary amplitude of the microwave oscillation, despite the presence of strong non-linearities. We propose here a nonlinear dynamics approach to investigate analytically the stability properties of OEOs. This approach predicts that the interplay of the delay and the intrinsic nonlinearity gives rise to unsuspected bifurcation-induced instabilities, in full agreement with our experimental results.

The OEO under study is organized in a single-loop architecture as depicted in Fig. 1. The oscillation loop consists of: $(i)$ A wideband integrated optics $\mathrm{LiNbO}_{3}$ Mach-Zehnder (MZ) modulator; it is seeded by a continuous-wave semiconductor laser of optical power $P$, which serves as a bifurcation parameter for scanning the OEO loop gain; the modulator is characterized by a half-wave voltage $V_{\pi}=4.2 \mathrm{~V}$, which defines the amplitude scale required at the microwave MZ driving voltage $V(t)$ for operation in the nonlinear regime. (ii) A thermalized $4 \mathrm{~km}$ fiber performs a time delay of $T=20 \mu \mathrm{s}$ on the microwave signal carried by the optical beam; the long delay is intended to support thousands of the microwave ringcavity modes; the free spectral range of $\Omega_{T} / 2 \pi=1 / T=50 \mathrm{kHz}$. (iii) A fast amplified photodiode with a conversion factor $S=2.2 \mathrm{~V} / \mathrm{mW}$. (iv) A narrow band microwave filter, intended to select the frequency range for the amplified modes; its central frequency is $\Omega_{0} / 2 \pi=3 \mathrm{GHz}$, and the $-3 \mathrm{~dB}$ bandwidth is $\Delta \Omega / 2 \pi=20 \mathrm{MHz} ;(v)$ A microwave amplifier with gain $G$ is closes the loop. All optical and electrical losses are gathered in a single attenuation factor $\eta$.

The dynamics of the microwave oscillation can therefore be described in terms of the dimensionless variable $x(t)=\pi V(t) / 2 V_{\pi}$ whose dynamics obeys [2]

$$
x+\tau \frac{d x}{d t}+\frac{1}{\theta} \int_{t_{0}}^{t} x(s) d s=\beta \cos ^{2}[x(t-T)+\phi]
$$

where $\beta=\pi \eta S G P / 2 V_{\pi}$ is the normalized loop gain, $\phi=\pi V_{B} / 2 V_{\pi}$ is the Mach-Zehnder offset phase, while $\tau=1 / \Delta \Omega$ and $\theta=\Delta \Omega / \Omega_{0}^{2}$ are the characteristic timescale parameters of the bandpass filter.

Assuming a monomode microwave oscillation of frequency $\Omega_{0}$ and complex amplitude $\mathcal{A}=|\mathcal{A}| e^{i \psi}$ for the variable $x(t)$, it is then possible to find an equation for the slowly- 
varying complex envelope amplitude, owing ton the fact that the nonlinear feedback term in Eq. (1) is reduced to the first order Bessel function $J_{1}$ through the Jacobi-Anger expansion $e^{i z \cos \alpha}=\sum_{n=-\infty}^{+\infty} i^{n} \mathrm{~J}_{\mathrm{n}}(z) e^{i n \alpha}$. Discarding the harmonics which are outside the bandwidth, we finally obtain the following delay-differential equation for the microwave complex envelope dynamics:

$$
\dot{\mathcal{A}}=-\mu \mathcal{A}-2 \mu \gamma e^{-i \sigma} \mathrm{Jc}_{1}\left[2\left|\mathcal{A}_{T}\right|\right] \mathcal{A}_{T}
$$

where $\gamma=\beta \sin 2 \phi$ is the effective loop gain, $\mu=\Delta \Omega / 2$ is the half-bandwidth of the filter, $\sigma=\Omega_{0} T$ is the round-trip phase shift of the microwave, while $\mathrm{Jc}_{1}$ is the Bessel cardinal function defined as $\mathrm{J}_{1}(x)=\mathrm{J}_{1}(x) / x$. Note that the Bessel cardinal function is qualitatively similar to the sinus cardinal function, but its absolute maximum is $1 / 2$ instead of 1 .

The advantage of dealing with an envelope equation is that the stationary states of the system are fixed points, which are solutions of $\mathcal{A}\left\{1+2 \gamma e^{i \sigma} \mathrm{Jc}_{1}[2|\mathcal{A}|]\right\}=0$. The phase matching condition $e^{i \sigma}= \pm 1$ has to be fulfilled for an oscillation to be sustained, and we here set $e^{i \sigma}=-1$ and $\gamma>0$ without loss of generality.

The trivial fixed point $\mathcal{A}(t) \equiv 0$ corresponds to the non-oscillating solution, and to check for its stability, we track the evolution of a perturbation $\delta \mathcal{A}$ with

$$
\delta \dot{\mathcal{A}}=-\mu \delta \mathcal{A}+\mu \gamma \delta \mathcal{A}_{T}
$$

A sufficient stability condition for this delayed variational equation is found to be $\gamma<1$.

At $\gamma=1$, a bifurcation occurs between the solution $\mathcal{A}(t)=0$ and the non-trivial fixed point $\mathcal{A}(t)=\mathcal{A}_{o} \neq 0$ which corresponds to the envelope of the rising microwave oscillation. From Eq. (2), it can be deduced that its amplitude obeys the equation $\mathrm{Jc}_{1}\left[2\left|\mathcal{A}_{o}\right|\right]=1 /(2 \gamma)$ which has a unique solution for $1<\gamma<15.52$.

Beyond the existence of this solution, we still have to check for its stability through the perturbation equation

$$
\begin{aligned}
\delta \dot{\mathcal{A}}= & -\mu \delta \mathcal{A}+2 \mu \gamma \\
& \times\left\{\mathrm{Jc}_{1}\left[2\left|\mathcal{A}_{o}\right|\right]+2\left|\mathcal{A}_{o}\right| \mathrm{Jc}_{1}^{\prime}\left[2\left|\mathcal{A}_{o}\right|\right]\right\} \delta \mathcal{A}_{T},
\end{aligned}
$$

whose stability is ensured for amplitudes $\left|\mathcal{A}_{o}\right|$ fulfilling the condition

$$
\left|\frac{1}{2}+\frac{\left|\mathcal{A}_{o}\right| \mathrm{Jc}_{1}^{\prime}\left[2\left|\mathcal{A}_{o}\right|\right]}{\mathrm{Jc}_{1}\left[2\left|\mathcal{A}_{o}\right|\right]}\right|<\frac{1}{2},
$$

corresponding to values of $\gamma$ belonging to the interval [1,2.31]. Therefore, the theory predicts that a pure single-mode solution emerges at $\gamma=1$, stable up to $\gamma_{c r}=2.31$. Beyond $\gamma_{c r}$, the system undergoes a supercritical Hopf bifurcation, as the fixed point $\mathcal{A}_{o}$ loses its stability while a limit cycle $\mathcal{A}_{o}+a_{o} \exp \left(i \Omega_{H} t\right)$ emerges. This Hopf bifurcation therefore leads to an 
amplitude modulation of the microwave signal $x(t)$, that is, to the emergence of deterministic modulation side-peaks in the radio-frequency Fourier spectrum. On the one hand, it can be demonstrated from the classical theory of Hopf bifurcations that the modulation amplitude $\left|a_{o}\right|$ initially grows as $\left[\gamma-\gamma_{c r}\right]^{1 / 2}$. On the other hand, it is also possible to determine analytically the frequency $\Omega_{H}$ of the Hopf-induced amplitude modulation. Effectively, the time-varying component $a_{o} \exp \left(i \Omega_{H} t\right)$ is initially very small and can be treated as a perturbation, which should obey Eq. (4): $\Omega_{H}$ is thus determined by the transcendental equation $\Omega_{H}=-\mu \tan \left[\Omega_{H} T\right]$, whose physical solution $\Omega_{H} \simeq \frac{1}{2} \Omega_{T}$ corresponds to a modulation period $T_{H}=2 T=40 \mu \mathrm{s}$.

Numerical simulations fully confirm the theoretical analysis. In Fig. 2, various timetraces obtained through the simulation of Eq. (2) are displayed. When $\gamma=2.2$, the system converges towards its stable fixed point, but only after some oscillatory transients. When the gain is further increased to $\gamma=2.4$, the system has yet undergone the supercritical Hopf bifurcation at $\gamma_{c r}=2.31$. As a consequence, the amplitude is modulated, and the modulation period is twice the delay-time as predicted. Numerical simulations are therefore in perfect agreement with the theory, both quantitatively and qualitatively.

The experimental evidence of this Hopf-induced amplitude modulation is presented in Fig. 3. Before the bifurcation, the amplitude is constant and there is a single peak in the microwave Fourier spectrum. Exactly at the onset of the bifurcation, the amplitude starts to be modulated with the Hopf frequency $\Omega_{H} / 2 \pi=\Omega_{T} / 4 \pi=25 \mathrm{kHz}$ : two modulation sidepeaks appear beside the carrier at the frequencies $\pm \Omega_{H} / 2 \pi$. Careful measurement of the corresponding critical value of the loop gain has given the experimental value of $\tilde{\gamma}_{c r}=2.42$, which is very near the analytical value $\gamma_{c r}=2.31$. Note that the lowest Hopf critical value was obtained after adjusting the MZ bias, most probably due to thermal drifts induced by the increasing RF power. After the bifurcation, the amplitude is strongly square-wave modulated with the same frequency $\Omega_{H}$, and the modulation side-peaks become stronger. This experimental phenomenology is therefore in perfect agreement with the analytically predicted scenario.

The bifurcation diagrams for the microwave variable $x(t)$ are displayed in Fig. 4. In fact, removing the periodic fast-scale oscillation at $\Omega_{0}$ is geometrically equivalent to represent the dynamics of a Poincaré section: therefore, at $\gamma=1$, the amplitude variable $\mathcal{A}(t)$ undergoes a pitchfork bifurcation (from the trivial fixed point to another fixed point) while the corresponding microwave variable $x(t)$ undergoes a Hopf bifurcation (from a fixed point to a limit-cycle); and at $\gamma=\gamma_{c r}, \mathcal{A}(t)$ undergoes a Hopf bifurcation while $x(t)$ undergoes a Neimark-Sacker bifurcation, that is, a bifurcation from a limit-cycle to a torus.

In conclusion, we have proposed a dynamical model for the study of single-mode optoelectronic oscillators. This model whose variable is the complex envelope amplitude of the 
microwave takes into account the intrinsic features of OEOs, which are a strong non-linearity on the one hand, and a very large delay on the other. As the loop gain is increased, the model predicts a supercritical Hopf bifurcation, that is, an amplitude modulation inducing the emergence of robust parasite side-peaks in the radio-frequency Fourier spectrum. The reported bifurcation phenomena has not been reported in the literature already published on the OEO, and we anticipate that many other phenomena might arise from this nonlinear dynamics approach, like for example multimode oscillations or phase noise stabilization/destabilization.

Extensions of this work are numerous. A particular interest of this model is also that it can easily be adapted to a wide class of oscillators derived from the OEOs, like coupled, dual-loop, tuneable or photonic filters OEOs. Along the same line, this modelling may improve the performance of these oscillators for other technological applications [3]. Finally, the principal interest of OEOs is their ultra-low phase noise $[4,5]$ : hence, it may be particularly interesting to derive a stochastic model of OEOs, based on the deterministic model we are proposing. For this purpose, if we consider a noisy gain $\gamma\left[1+\xi_{m}(t)\right]$ and an additive noise term $\xi_{a}(t)$ in Eq. (2), we can derive a stochastic differential equation for the phase $\psi(t)$ of the microwave. This would be an interesting challenge that would couple a new theoretical problem to a plethora of applications.

Y. K. C. acknowledges a research grant from the Govern de les Illes Balears. Financial support from EC project PICASSO IST-2005-34551, MEC (Spain) and FEDER project TEC2006-1009/MIC (PhoDECC) is also acknowledged.

\section{References}

1. X. S. Yao and L. Maleki, "High frequency optical subcarrier generator", Electron. Lett. 30, 1525 (1994).

2. N. Gastaud, S. Poinsot, L. Larger, M. Hanna, J.-M. Merolla, J.-P. Goedgebuer, and F. Malassenet, "Electro-Optical Chaos for 10 GBit/s Optical Transmissions", Electron. Lett. 40, 898 (2004).

3. J. Lasri, P. Devgan, R. Tang and P. Kumar, "Ultralow timing jitter 40-Gb/s clock recovery using a self-starting optoelectronic oscillator", IEEE Photon. Technol. Lett., 16, 263 (2004).

4. X. S. Yao and L. Maleki, "Optoelectronic microwave oscillator", J. Opt. Soc. Am. B. 13, 1725 (1996).

5. E. Rubiola and V. Giordano, "Advanced interferometric phase and amplitude noise measurements", Rev. Sci. Instr. 73, 2445 (2002). 


\section{List of Figure Captions}

Fig. 1. Experimental set-up.

Fig. 2. Numerical simulations of Eq. (2), for various values of the effective feedback gain $\gamma$, with $\sigma=\pi$ and $\phi=\pi / 4$. a) $\gamma=2.2<\gamma_{c r}$ : the amplitude converges to a constant value. $b$ ) $\gamma=2.4>\gamma_{c r}$ : the amplitude is modulated with a period equal to $2 T=40 \mu$ s.

Fig. 3. Experimental evidence of the Hopf-induced amplitude modulation, as the gain is increased; $a 1, b 1$ and $c 1$ are timetraces, and $a 2, b 2$ and $c 2$ are the Fourier spectra of the corresponding reconstructed envelopes (relatively to the carrier at $\Omega_{0} / 2 \pi=3 \mathrm{GHz}$ ). $a$ ) Before the bifurcation. b) At the onset of the bifurcation. c) After the bifurcation.

Fig. 4. Bifurcation diagrams for the microwave variable $x(t)$ revealing unexpected nonlinear effects (to be compared with Fig. 4 in ref. [4]). a) Theoretical. b) Experimental. 


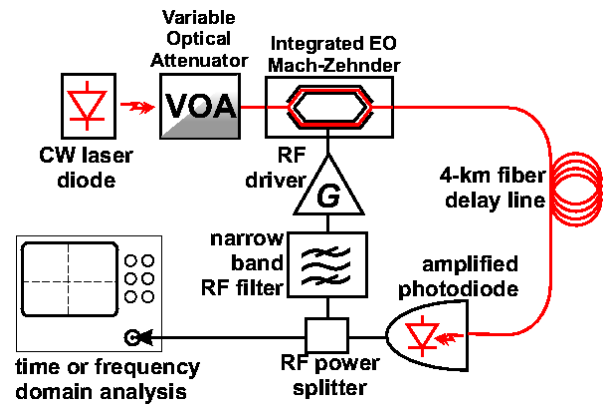

Fig. 1. Experimental set-up. Fig1.eps 


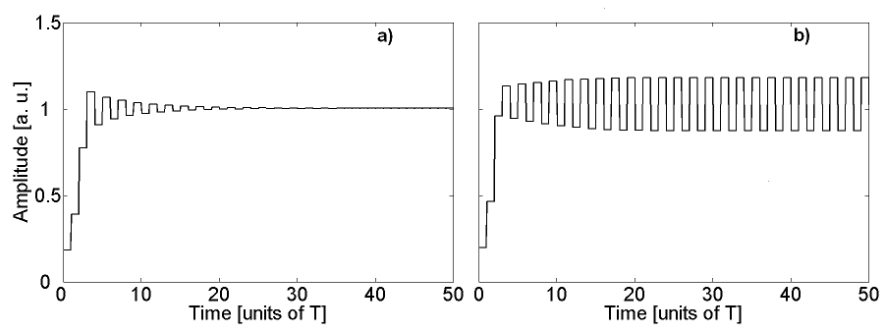

Fig. 2. Numerical simulations of Eq. (2), for various values of the effective feedback gain $\gamma$, with $\sigma=\pi$ and $\phi=\pi / 4$. a) $\gamma=2.2<\gamma_{c r}$ : the amplitude converges to a constant value. $b) \gamma=2.4>\gamma_{c r}$ : the amplitude is modulated with a period equal to $2 T=40 \mu$ s. Fig2.eps 

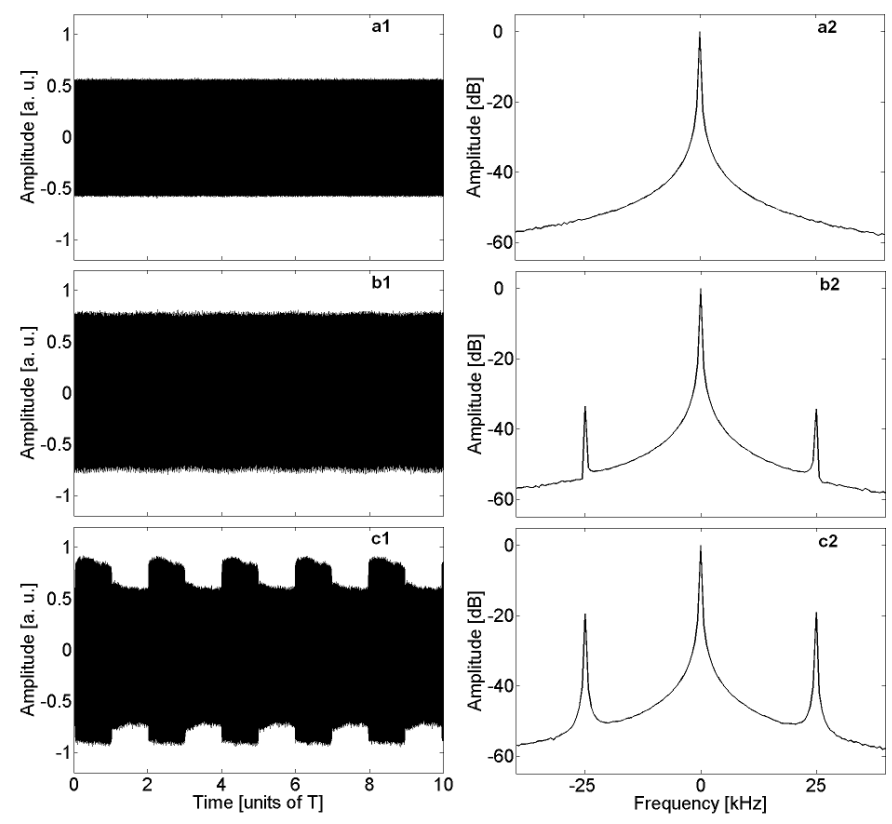

Fig. 3. Experimental evidence of the Hopf-induced amplitude modulation, as the gain is increased; $a 1, b 1$ and $c 1$ are timetraces, and $a 2, b 2$ and $c 2$ are the Fourier spectra of the corresponding reconstructed envelopes (relatively to the carrier at $\Omega_{0} / 2 \pi=3 \mathrm{GHz}$ ). a) Before the bifurcation. b) At the onset of the bifurcation. c) After the bifurcation. Fig3.eps 


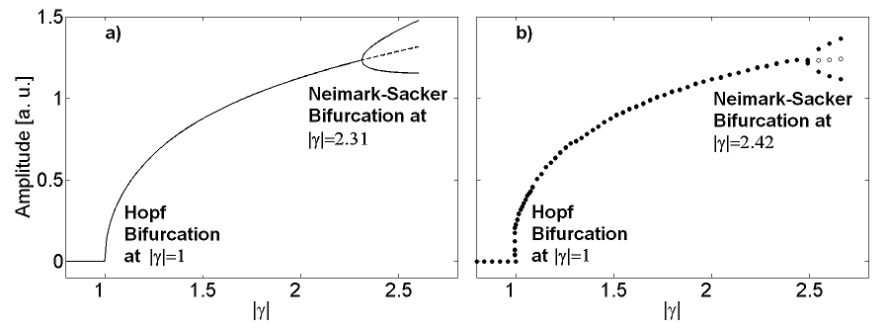

Fig. 4. Bifurcation diagrams for the microwave variable $x(t)$ revealing unexpected nonlinear effects (to be compared with Fig. 4 in ref. [4]). a) Theoretical. b) Experimental. Fig4.eps 